This leads to the following result: The quasi-elastic axes of the system are the lines of the regulus the directrices of which are $e_{k}+\epsilon e_{k} \cdot \Gamma, k=1,2,3$.

The traces of the directrices $e_{k}+\epsilon e_{k} \cdot \Gamma$ on the principal planes have been called elastic centers. The locus of an elastic center starts, with $\omega=0$, at a point that depends on the parameters of the elastic suspension and ends at the center of gravity $(\omega=\infty)$.

In the conservative case this locus is the outside section of a hyperbola, the inside section corresponding to $\omega^{2}<0$. The locus is a 4 th degree algebraic curve in the nonconservative case. The reduced system in which one reaction goes through the center of gravity and the other two lie in a plane through this center has been considered in detail. ${ }^{5}$

\title{
HEAVY DISK SUPPORTED BY CONCENTRATED FORCES*
}

BY YI-YUAN YU (Washington University, St. Louis, Mo.)

Muschelišvili solved the problem of a two-dimensional light disk subjected to an arbitrary number of concentrated forces by means of his method of complex variable $[1,273-274] .^{* *}$ When the weight of the disk has to be taken into consideration, the problem may still be solved in a similar way. Muschelišvili's notations will be followed throughout this paper, and only additional ones will be defined as they first occur.

In plane problems including body forces due to gravity, the stress function $U$ may still satisfy the biharmonic equation if it is defined by the following equations:

$$
\frac{\partial^{2} U}{\partial x^{2}}=\tau_{\nu y}-V_{1}, \quad \frac{\partial^{2} U}{\partial y^{2}}=\tau_{x x}-V_{1}, \quad-\frac{\partial^{2} U}{\partial x \partial y}=\tau_{x y}
$$

in which $V_{1}$ is the body force potential due to gravity and is equal to $w y$ when gravity acts in the negative $y$-direction [3], $w$ being the specific weight of the material of the body. Hence, the function $U$ may be expressed in terms of two analytic functions as shown by Muschelišvili [2, 284].

The boundary conditions

$$
\tau_{x}=\tau_{x x} \frac{d y}{d s}-\tau_{x y} \frac{d x}{d s}, \quad \tau_{y}=\tau_{x y} \frac{d y}{d s}-\tau_{y y} \frac{d x}{d s}
$$

however, may be shown to lead to some different result. When stress components given by Eqs. (1) are substituted into these conditions and computations carried out in the same manner as given by Muschelišvili [2, 301-302], the following result is obtained:

$$
\varphi_{1}(z)+z \bar{\varphi}_{1}^{\prime}(\bar{z})+\bar{\psi}_{1}(\bar{z})=i \int\left(\tau_{x}+i \tau_{y}\right) d s-\int V_{1} d z
$$

If we define

$$
f_{1}+i f_{2}=i \int\left(\tau_{x}+i \tau_{y}\right) d s-\int V_{1} d z
$$

${ }^{5} \mathrm{R} . \mathrm{K}$. Bernhard and J. J. Slade, Jr., On the elastic center of one-mass plane oscillatory systems (unpublished). Dynamics Laboratory, Bureau of Engineering Research, Rutgers University.

*Received October 15, 1951.

**The first number in each square bracket refers to the References listed at the end of the paper. The subsequent numbers, if any, refer to the page numbers of the reference. 
then the boundary equation for the first fundamental boundary value problem is

$$
\varphi_{1}(z)+z \bar{\varphi}_{1}^{\prime}(\bar{z})+\bar{\psi}_{1}(\bar{z})=f_{1}+i f_{2}
$$

which holds true on the boundary $C$ of the body in the original $z$-plane. When $C$ is mapped into the unit circle $\gamma$ in the $\zeta$-plane by means of the function $z=\omega(\zeta)$, the boundary equation becomes

$$
\varphi(\sigma)+\frac{\omega(\sigma)}{\bar{\omega}^{\prime}(\bar{\sigma})} \bar{\varphi}^{\prime}(\bar{\sigma})+\bar{\psi}(\bar{\sigma})=f_{1}+i f_{2} \quad(\text { on } \gamma)
$$

Thus, except with a different definition of $f_{1}+i f_{2}$, this equation assumes the same form as the one for zero body forces [2, 294].

Modified expressions for stress and displacement components may similarly be derived. Only stress components in curvilinear coordinates are given here:

$$
\left.\begin{array}{rl}
\tau_{\rho \rho}+\tau_{\theta \theta} & =2[\Phi(\zeta)+\bar{\Phi}(\bar{\zeta})]+2 V(\zeta) \\
\tau_{\theta \theta}-\tau_{\rho \rho}+2 i \tau_{\rho \theta} & =\frac{2 \zeta^{2}}{\rho^{2} \bar{\omega}^{\prime}(\bar{\zeta})}\left[\bar{\omega}(\bar{\zeta}) \Phi^{\prime}(\zeta)+\omega^{\prime}(\zeta) \Psi(\zeta)\right]
\end{array}\right\}
$$

in which

$$
V(\zeta)=V_{1}(\omega(\zeta))=V_{1}(z)
$$

By comparing with Muschelišvili's original formulas [2,312], it can be seen that $2 V(\zeta)$ is the only additional term due to body forces.

The problem here concerned is that of a heavy disk having radius $R$ supported by an arbitrary number of, say $n$, concentrated forces $\left(X_{1}, Y_{1}\right),\left(X_{2}, Y_{2}\right), \cdots,\left(X_{n}, Y_{n}\right)$ at points on its boundary corresponding to $\sigma_{1}, \sigma_{2}, \cdots, \sigma_{n}$ respectively on the unit circle as shown in the figure. Obviously the supporting forces must satisfy the following conditions:

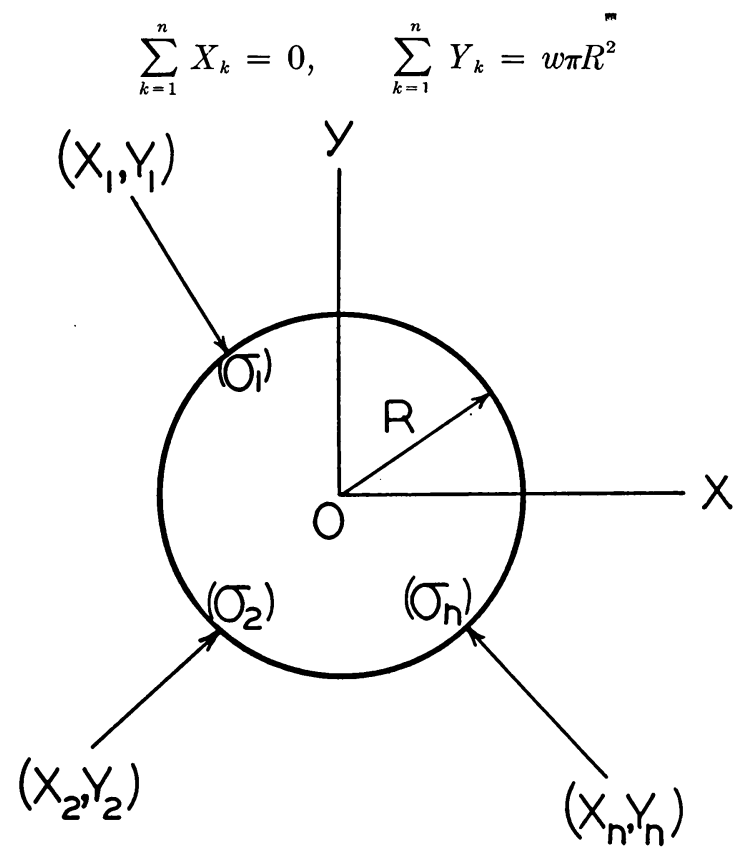


Since the boundary of the disk is free from stress everywhere except at the supporting points, expression (2) becomes

$$
f_{1}+i f_{2}=-\int V_{1} d z=\frac{1}{2} i w \int(z-\bar{z}) d z
$$

For a circle of radius $R$, we have

$$
z=\omega(\zeta)=R \zeta
$$

hence,

$$
f_{1}+i f_{2}=\frac{1}{2} i w \int R^{2}(\sigma-\bar{\sigma}) d \sigma=\frac{1}{2} i w R^{2}\left(\frac{\sigma^{2}}{2}-\log \sigma\right)
$$

The boundary equation (3) now takes the form

$$
\varphi(\sigma)+\sigma \bar{\varphi}^{\prime}(\bar{\sigma})+\bar{\psi}(\bar{\sigma})=\frac{1}{2} i w R^{2}\left(\frac{\sigma^{2}}{2}-\log \sigma\right) \quad(\text { on } \gamma)
$$

By modifying those obtained by Muschelišvili for the problem of a light disk [1, 274], the two analytic functions for the present problem can be written down as

$$
\left.\begin{array}{l}
\varphi(\zeta)=-\frac{1}{2 \pi} \sum_{k=1}^{n}\left(X_{k}+i Y_{k}\right) \log \left(\sigma_{k}-\zeta\right)+\varphi^{0}(\zeta) \\
\psi(\zeta)=\frac{1}{2 \pi} \sum_{k=1}^{n}\left(X_{k}-i Y_{k}\right) \log \left(\sigma_{k}-\zeta\right)-\frac{1}{2 \pi} \sum_{k=1}^{n} \frac{\left(X_{k}+i Y_{k}\right) \bar{\sigma}_{k}}{\sigma_{k}-\zeta}+\psi^{0}(\zeta)
\end{array}\right\}
$$

in which $\varphi^{0}(\zeta)$ and $\psi^{0}(\zeta)$ are functions analytic in the entire region inside $\gamma$ and have the forms $[1,272]$

$$
\begin{aligned}
& \varphi^{0}(\zeta)=\alpha_{1} \zeta+\left(\alpha_{2}+i \beta_{2}\right) \zeta^{2}+\cdots \\
& \psi^{0}(\zeta)=\alpha_{0}^{\prime}+i \beta_{0}^{\prime}+\left(\alpha_{1}^{\prime}+i \beta_{1}^{\prime}\right) \zeta+\left(\alpha_{2}^{\prime}+i \beta_{2}^{\prime}\right) \zeta^{2}+\cdots
\end{aligned}
$$

The other terms in $\varphi(\zeta)$ and $\psi(\zeta)$ account for the singularities in the solution due to concentrated forces and therefore have the same forms as those for a light disk.

Substituting expressions (6) into Eq. (5) now yields, after simplification,

$$
\varphi^{0}(\sigma)+\sigma \bar{\varphi}^{-0 \prime}(\bar{\sigma})+\bar{\psi}^{0}(\bar{\sigma})=\frac{i w R^{2}}{4} \sigma^{2}-\frac{1}{2 \pi} \sum_{k=1}^{n}\left(X_{k}-i Y_{k}\right) \sigma_{k} \sigma
$$

from which $\varphi^{0}(\zeta)$ and $\psi^{0}(\zeta)$ can be determined. Following the established procedure of the method, we formulate an integral equation by multiplying Eq. (7) through by $1 / 2 \pi i d \sigma /(\sigma-\zeta)$ and integrating around $\gamma$. The integrals in the equation thus obtained can be evaluated by means of the theorems developed by Muschelišvili [1, 269]. The result gives

$$
\varphi^{9}(\zeta)+\alpha_{1} \zeta+2\left(\alpha_{2}-i \beta_{2}\right)+\alpha_{0}^{\prime}-i \beta_{0}^{\prime}=\frac{i w R^{2}}{4} \zeta^{2}-\frac{1}{2 \pi} \sum_{k=1}^{n}\left(X_{k}-i Y_{k}\right) \sigma_{k} \zeta
$$

in which the constant terms may be neglected. The constant $\alpha_{1}$ is determined by differ- 
entiating the rest of the equation with respect to $\zeta$ once and setting $\zeta$ equal to zero. We have finally

$$
\varphi^{0}(\zeta)=\frac{i w R^{2}}{4} \zeta^{2}-\frac{1}{4 \pi} \sum_{k=1}^{n}\left(X_{k}-i Y_{k}\right) \sigma_{k} \zeta
$$

Substituting this back into Eq. (7) and formulating the conjugate of the result, we obtain

$$
\psi^{0}(\sigma)=-\frac{1}{2} i w R^{2}+\frac{1}{4 \pi} \sum_{k=1}^{n}\left[\left(X_{k}-i Y_{k}\right) \sigma_{k}-\left(X_{k}+i Y_{k}\right) \bar{\sigma}_{k}\right] \bar{\sigma}
$$

Multiplying this through by $1 / 2 \pi i d \sigma /(\sigma-\zeta)$ and integrating around $\gamma$,

$$
\psi^{0}(\zeta)=-\frac{1}{2} i w R^{2}
$$

Thus the problem is completely solved. The solution consists of $\varphi(\zeta)$ and $\psi(\zeta)$ as given by $(6)$, and $\varphi^{\circ}(\zeta)$ and $\psi^{\circ}(\zeta)$ are given respectively by $(8)$ and $(9)$.

The problem of a heavy disk resting on a horizontal plane was solved by $\mathrm{J}$. H. Michell [4] and represents a special case of our problem in which

$$
n=1, \quad X_{1}=0, \quad Y_{1}=w \pi R^{2}, \quad \sigma_{1}=-i
$$

The analytic functions reduce to

$$
\begin{aligned}
& \varphi(\zeta)=-\frac{i w R^{2}}{2} \log (\zeta+i)+\frac{i w R^{2}}{4} \zeta^{2}+\frac{w R^{2}}{4} \zeta \\
& \psi(\zeta)=-\frac{w R^{2}}{2} \frac{1}{\zeta+i}-\frac{i w R^{2}}{2} \log (\zeta+i)-\frac{1}{2} i w R^{2}
\end{aligned}
$$

The sum of the normal stress components can be computed according to the first of Eqs. (4); thus,

$$
\tau_{\rho \rho}+\tau_{\theta \theta}=w R\left(1-2 \frac{\rho \sin \theta+1}{\rho^{2}+2 \rho \sin \theta+1}\right)
$$

It can readily be shown that both normal stress components vanish at all points on the boundary of the disk except the point of support.

The problem of a heavy disk resting on the ends of its horizontal diameter, as was recently solved by Horvay and Poritsky [5], is another special case in which

$$
n=2, \quad X_{1}=X_{2}=0, \quad Y_{1}=Y_{2}=\frac{1}{2} w \pi R^{2}, \quad \sigma_{1}=1, \quad \sigma_{2}=-1
$$

The analytic functions are

$$
\begin{gathered}
\varphi(\zeta)=-\frac{i w R^{2}}{4} \log \left(\zeta^{2}-1\right)+\frac{i w R^{2}}{4} \zeta^{2} \\
\psi(\zeta)=\frac{i w R^{2}}{2} \frac{1}{\zeta^{2}-1}-\frac{i w R^{2}}{4} \log \left(\zeta^{2}-1\right)-\frac{1}{2} i w R^{2}
\end{gathered}
$$

The normal stress components at any point on the boundary of the disk except the two at the supports are given by

$$
\left(\tau_{\rho \rho}\right)_{\rho=1}=0, \quad\left(\tau_{\theta \theta}\right)_{\rho=1}=-\frac{w R}{\sin \theta}
$$




\title{
REFERENCES
}

1. N. Muschelišvili, Praktische Lösung der fundamentalen Randwertaufgaben der Elastizitätstheorie in der Ebene für einige Berandungsformen, ZaMM 13, 264-282 (1933).

2. N. Muschelišvili, Recherches sur les problèmes aux limites relatifs à l'équation biharmonique et aux equations de l'élasticité à deux dimensions, Math. Annalen 107, 282-312 (1933).

3. S. Timoshenko, Theory of elasticity, McGraw-Hill, New York, 1934, pp. 25-26.

4. H. Love, Mathematical theory of elasticity, 4th ed., 1927, p. 219.

5. G. Horvay and H. Poritsky, Gravitational stresses in a disk supported at the ends of the horizontal diameter, General Electric, Knolls Atomic Power Lab., Schenectady, N. Y., 1951.

\section{REPRESENTATION OF NONLINEAR FIELD FUNCTIONS BY THIELE SEMI-INVARIANTS ${ }^{1}$}

\author{
BY WILLIAM M. MACDONALD, III, ${ }^{2}$ JOHN M. RICHARDSON, ${ }^{3}$ \\ AND LEON P. ROSENBERRY ${ }^{4}$
}

1. Certain nonlinear field functions, possessing the property of space localization of the gradients of the dependent variables, occur in the problem of flame propagation in continuous media. The difficulties encountered in solving the equations of propagation may be considerably diminished by introducing new dependent variables (intrinsically connected with this localization) within a certain region, outside of which the equations may be linearized and treated in a point-wise sense by well-known methods. Specifically, we choose the $x$-axis as nearly perpendicular to the flame front and define the region as $x_{1}(y, z) \leqq x \leqq x_{2}(y, z)$. Taking a state variable, temperature $T$, for example, we choose the new dependent variables as the $\kappa_{\nu}$ given by

$$
\sum_{\nu=0}^{\infty}(i t)^{\nu} \frac{\kappa_{\nu}}{\nu !}=\log [(\phi t)]
$$

where

$$
\phi(t)=\int_{x_{1}}^{x_{2}} d x e^{i t x} \frac{\partial T}{\partial x}
$$

These definitions are closely related to the formalism of mathematical statistics. In particular, if one considers $\partial T / \partial x$ to correspond to an unnormalized distribution function with range $\left(x_{1} \leqq x \leqq x_{2}\right)$, the $\kappa_{\nu}$ correspond to the Thiele semi-invariants [1] of $\partial T / \partial x$ and completely describe a given function in the range $\left(x_{1} \leqq x \leqq x_{2}\right)$.

Received Oct. 8, 1951.

${ }^{1}$ This research is part of the work being done by the Bureau of Mines on Contract NA onr 25-47, NR 090 117, (33-038) 51-4151, supported by the Office of Naval Research and the Air Materiel Command.

${ }^{2}$ Formerly Physicist, Mathematical and Theoretical Physics Section, Explosives and Physical Sciences Division, Bureau of Mines, Pittsburgh, Pa. Present åddress: Department of Physics, Princeton University.

${ }^{3}$ Head, Kinetics Section, Explosives and Physical Sciences Division, Bureau of Mines, Pittsburgh, Pa.

${ }^{4}$ Physicist, Mathematical and Theoretical Physics Section, Explosives and Physical Sciences Division, Pittsburgh, Pa. 